\title{
Sporadic human Yersinia enterocolitica infections caused by bioserotype 4/O:3 originate mainly from pigs
}

\author{
Maria Fredriksson-Ahomaa, ${ }^{1} \dagger$ Andreas Stolle, ${ }^{1}$ Anja Siitonen ${ }^{2}$ \\ and Hannu Korkeala ${ }^{3}$ \\ ${ }^{1}$ Institute of Hygiene and Technology of Food of Animal Origin, Ludwig-Maximilian University, \\ Munich, Germany \\ ${ }^{2}$ Department of Bacterial and Inflammatory Diseases, National Public Health Institute (KTL), \\ Helsinki, Finland \\ ${ }^{3}$ Department of Food and Environmental Hygiene, University of Helsinki, Finland
}

Received 16 January 2006

Accepted 13 February 2006
Yersinia enterocolitica $4 / 0: 3$ is the most frequent cause of sporadic human yersiniosis in Finland and Germany. To investigate the possible link between pigs and humans, 282 human and 534 porcine strains from Finland and Germany were characterized with PFGE using Notl, Apal and Xhol enzymes. Most of the human strains ( $>80 \%$ ) were indistinguishable from the porcine strains in both countries and most of the genotypes (178/182) were different in Finland and Germany. The indistinguishable genotypes among human and porcine strains together with different genotypes in Finland and Germany indicate that pigs are an important source of sporadic yersiniosis in both countries.

\section{INTRODUCTION}

Yersinia enterocolitica is a common food-borne pathogen causing yersiniosis in humans (Bottone, 1997). In addition to acute gastroenteritis, the infection may lead to various post-infectious complications such as reactive arthritis. In 2004 , the incidence of human yersiniosis was $13 \cdot 1$ and $7 \cdot 5$ per 100000 inhabitants in Finland (Anonymous, 2005a) and Germany (Anonymous, 2005b), respectively, and the most frequently found pathogenic type was the bioserotype 4/ $\mathrm{O}: 3$. Pigs are the only reservoir from which $Y$. enterocolitica 4/O :3 strains have been frequently isolated. The prevalence of this bioserotype in slaughter pigs has been reported to be $56 \%$ in Finland (Korte et al., 2004) and $60 \%$ in southern Germany (Fredriksson-Ahomaa et al., 2001a).

Despite the fact that $Y$. enterocolitica is an important foodborne pathogen, pathogenic strains have seldom been isolated from food, except from edible pig offal (tongue, heart, liver and kidneys). However, this might be more of a methodological problem, since higher detection rates have been obtained from raw pork investigated by PCR than by conventional culture methods (Fredriksson-Ahomaa \& Korkeala, 2003). Yersiniosis was associated with eating raw or undercooked pork and drinking untreated water in the 2 weeks before illness in some case-control studies

†Present address: Institute of Hygiene and Technology of Food of Animal Origin, Schoenleutnerstr. 8, D-85764 Oberschleissheim, Germany.
(Tauxe et al., 1987; Ostroff et al., 1994). No association was observed with eating eggs, beef or vegetables, or with drinking milk (Tauxe et al., 1987). In a case-control study, yersiniosis clearly was associated with chitterlings (boiled pig small intestines) (Jones et al., 2003).

Several phenotypic and genotypic methods have been used to characterize $Y$. enterocolitica strains from various sources (Kapperud et al., 1990; Iteman et al., 1996). While human strains were shown to be indistinguishable from the porcine strains using restriction endonuclease analysis of chromosomal or plasmid DNA, the discriminatory power of $Y$. enterocolitica $4 / \mathrm{O}: 3$ with these two methods was low, the discriminatory indices being only 0.623 and $0 \cdot 174$, respectively (Kapperud et al., 1990). Genetic diversity is limited among strains belonging to bioserotype $4 / \mathrm{O}: 3$ but, with PFGE using NotI, ApaI and XhoI enzymes, this group can be divided efficiently into several genotypes with a discriminatory index of 0.93 (Fredriksson-Ahomaa et al., 1999). Amplified fragment length polymorphism is a recently adopted typing method that could be an alternative method in parallel with PFGE to elucidate the epidemiology of $Y$. enterocolitica 4/O:3 (Fearnley et al., 2005).

As in other sporadic infections, the infection routes of sporadic yersiniosis are also difficult to trace. So far, a clear link between pigs and humans has not been demonstrated. In order to study this possible link, a molecular epidemiological approach was used to compare Finnish and German genotypes of $Y$. enterocolitica 4/O:3 strains isolated from 
humans with yersiniosis and from porcine sources with each other.

\section{METHODS}

A total of $816 Y$. enterocolitica $4 / \mathrm{O}: 3$ strains isolated from faecal specimens of humans with diarrhoea in Finland $(n=212)$ and Bavaria $(n=70)$ and from different porcine sources in Finland $(n=394)$ and Bavaria $(n=140)$ from 1995 to 2003 was investigated. The porcine strains were recovered from samples collected from slaughterhouses, including faeces, carcass, tonsil, tongue, diaphragm, heart, kidney and liver samples, and from retail outlets, including tongue, heart, kidney, liver and pork samples. All 282 human and 534 porcine strains were characterized with PFGE using NotI, ApaI and $\mathrm{XhoI}$ rare-cutting restriction enzymes, as described previously (Fredriksson-Ahomaa et al., 1999). The genotypes were considered to be different if any band difference in fragments over $70 \mathrm{~kb}$ was observed.

\section{RESULTS AND DISCUSSION}

Overall, 182 different genotypes (GTs) of Y. enterocolitica 4/O :3 were found with PFGE using NotI, ApaI and XhoI: 138 (GT1-GT138) among the 606 Finnish strains and 48 (GT1, GT136-GT182) among the 210 German strains (Table 1). Of the Finnish human strains, $81 \%$ (171/212) belonging to 40 genotypes (GT1-GT39, GT137) were indistinguishable from porcine strains isolated in Finland. Correspondingly, $83 \%(58 / 70)$ of the Bavarian human strains belonging to 13 genotypes (GT136-GT148) were indistinguishable from porcine strains isolated in Bavaria. These data strongly support the hypothesis that pigs play an important role in the epidemiology of human sporadic $Y$. enterocolitica 4/O:3 infections in Finland and Germany. Furthermore, they also show that biodiversity among the strains of porcine origin is higher than that among human strains in both countries. Seventy genotypes (GT66-GT135) containing $33 \%(130 / 394)$ of the porcine strains in Finland and 26 genotypes (GT157-GT182) containing 35\% (49/ 140) of the porcine strains in Bavaria were not recovered from humans (Table 1). One reason may be that the strains of these genotypes are less virulent and thus cause human infection less frequently. Another reason may be that the prevalence of these genotypes is low and therefore they were not present among the 282 human strains tested.

Surprisingly, $98 \%$ (178/182) of the genotypes (GT2-GT135, GT139-GT182) were different in Finland and southern Germany (Table 1). Furthermore, Y. enterocolitica 4/O:3 strains isolated from pig tonsils in Bavaria have been shown to belong to different genotypes to strains isolated from the same source in Finland (Fredriksson-Ahomaa et al., 2003), indicating a differential geographical distribution of Bavarian and Finnish genotypes. Thus it seems that distinct Y. enterocolitica populations have developed in Finland and Bavaria. A natural explanation might be that no importation of live farm animals into Finland was allowed prior to 1995. Because of the great geographical distance between Finland and southern Germany, even today live pigs are not transported between these countries.

Only four genotypes (GT1, GT136-138) were found in both Finland and southern Germany. GT1 was the most common type among Finnish strains isolated from humans and porcine sources (Table 1). The same type was found only once among German human strains. GT136 and GT137 were the most common types among German strains isolated from humans and porcine sources (Table 1), whereas GT136 was found only twice among human strains in Finland, and only one human and porcine strain was GT137. GT138 was recovered from both human and porcine sources

Table 1. Distribution of 182 genotypes among $816 Y$. enterocolitica $4 / O: 3$ strains isolated in Finland and southern Germany

\begin{tabular}{|c|c|c|c|c|c|c|}
\hline \multirow{2}{*}{$\begin{array}{l}\text { Genotypes } \\
\text { (GT1-GT182) }\end{array}$} & \multicolumn{3}{|c|}{ Finland $^{\star}$} & \multicolumn{3}{|c|}{ Bavaria } \\
\hline & $\begin{array}{c}\text { Total } \\
(n=606)\end{array}$ & $\begin{array}{l}\text { Human } \\
(n=212)\end{array}$ & $\begin{array}{l}\text { Porcine } \\
(n=394)\end{array}$ & $\begin{array}{c}\text { Total } \\
(n=210)\end{array}$ & $\begin{array}{l}\text { Human } \\
(n=70)\end{array}$ & $\begin{array}{l}\text { Porcine } \\
(n=140)\end{array}$ \\
\hline $1 \dagger$ & 123 & 47 & 76 & 1 & 1 & 0 \\
\hline $2-39 \ddagger$ & 310 & 123 & 187 & 0 & 0 & 0 \\
\hline $40-65$ & 37 & 37 & 0 & 0 & 0 & 0 \\
\hline $66-135$ & 130 & 0 & 130 & 0 & 0 & 0 \\
\hline $136 \$$ & 2 & 2 & 0 & 43 & 21 & 22 \\
\hline $137 \S$ & 2 & 1 & 1 & 40 & 17 & 23 \\
\hline 138 & 2 & 2 & 0 & 8 & 1 & 7 \\
\hline 139-148‡ & 0 & 0 & 0 & 58 & 19 & 39 \\
\hline 149-156 & 0 & 0 & 0 & 11 & 11 & 0 \\
\hline $157-182$ & 0 & 0 & 0 & 49 & 0 & 49 \\
\hline
\end{tabular}

${ }^{\star}$ Some of these results have been reported previously by Fredriksson-Ahomaa et al. (2001b).

$\dagger$ The most common genotype among Finnish strains isolated from human and porcine sources.

$\ddagger$ The total prevalence of each genotype in these groups was $\leqslant 7 \%$.

\$The two most common genotypes among German strains isolated from human and porcine sources. 
Table 2. Porcine sources of human genotypes of Yersinia enterocolitica $4 / 0: 3$ strains found in Finland and southern Germany

\begin{tabular}{|crcl}
\hline Country & GT & $\begin{array}{c}\text { No. of porcine } \\
\text { strains }\end{array}$ & \multicolumn{1}{c|}{ Porcine sources (no. of strains) } \\
\hline \multirow{2}{*}{ Finland } & $1^{*}$ & 76 & Faeces (1), carcass (19), tonsils (16), tongue (24), heart (2), liver (3), kidney (6), pork (5) \\
& $2-39$ & 187 & Faeces (1), carcass (8), tonsils (58), tongue (81), heart (9), liver (9), kidney (14), pork (7) \\
& 137 & 1 & Pork (1) \\
Bavaria & $136 \dagger$ & 22 & Tonsils (5), tongue (1), lungs (3), diaphragm (1), pork (12) \\
& $137 \dagger$ & 23 & Tonsils (9), tongue (1), diaphragm (1), lungs (2), heart (3), liver (1), pork (6) \\
& $138-148$ & 46 & Faeces (1), tonsils (14), tongue (6), diaphragm (3), lungs (3), heart (5), liver (1), \\
& & & kidneys (2), pork (11)
\end{tabular}

${ }^{\star}$ The most common genotype among Finnish human strains.

$\dagger$ The two most common genotypes among German human strains.

in southern Germany, but only two human strains in Finland were GT138. One reason for the indistinguishable genotypes (GT1, GT136-GT138) among some human strains in Finland and Germany may be the ever-increasing level of travel. However, it is more likely that the vast majority of the human $Y$. enterocolitica $4 / \mathrm{O}: 3$ infections were acquired domestically.

The 40 Finnish human genotypes GT1-GT39 and GT137 and 13 German human genotypes GT136-GT148 were isolated from several different samples of porcine origin collected from slaughterhouses and retail outlets (Table 2). Most Finnish genotypes (39/40) differed from the 13 Bavarian genotypes, only genotype GT137 being found in both Finland and Bavaria. This genotype was found in several porcine samples in southern Germany, but only once from a pork sample in Finland (Table 2). An explanation may be that most of the pork consumed is from the domestic market. However, it is probable that more indistinguishable strains will be found worldwide in the future as a result of the steadily increasing international trade in foodstuffs.

The indistinguishable genotypes among human and porcine strains together with different genotypes in Finland and southern Germany, the high prevalence of Y. enterocolitica 4/O:3 among pigs and contamination of pork with $Y$. enterocolitica $4 / \mathrm{O}: 3$ strongly indicate that pigs are the primary source of sporadic human $Y$. enterocolitica $4 / O: 3$ infection in Finland and southern Germany. Possible transmission routes from pigs to humans are directly from pigs, via contaminated pork products or indirectly from pets that have been fed raw pork (Fredriksson-Ahomaa et al., 2001b).

\section{REFERENCES}

Anonymous (2005a). Infectious Disease in Finland 2004. KTL B12/ 2005. Helsinki, Finland: National Public Health Institute.

Anonymous (2005b). Infektionsepidemiologisches Jahrbuch Meldepflichtiger Krankheiten für 2004. Berlin, Germany: Robert Koch Institute.

Bottone, E. J. (1997). Yersinia enterocolitica: the charisma continues. Clin Microbiol Rev 10, 257-276.
Fearnley, C., On, S. L. W., Kokotovic, B., Manning, G., Cheasty, T. \& Newell, D. G. (2005). Application of fluorescent amplified fragment length polymorphism for comparison of human and animal isolates of Yersinia enterocolitica. Appl Environ Microbiol 71, 4960-4965.

Fredriksson-Ahomaa, M. \& Korkeala, H. (2003). Low occurrence of pathogenic Yersinia enterocolitica in clinical, food and environmental samples: a methodological problem. Clin Microbiol Rev 16, 220-229.

Fredriksson-Ahomaa, M., Autio, T. \& Korkeala, H. (1999). Efficient subtyping of Yersinia enterocolitica bioserotype 4/O:3 with pulsedfield gel electrophoresis. Lett Appl Microbiol 29, 308-312.

Fredriksson-Ahomaa, M., Bucher, M., Hank, C., Stolle, A. \& Korkeala, H. (2001a). High prevalence of Yersinia enterocolitica 4:O3 on pig offal in southern Germany: a slaughtering technique problem. Syst Appl Microbiol 24, 457-463.

Fredriksson-Ahomaa, M., Hallanvuo, S., Korte, T., Siitonen, A. \& Korkeala, H. (2001b). Correspondence of genotypes of sporadic Yersinia enterocolitica bioserotype 4/O:3 strains of human and porcine sources. Epidemiol Infect 127, 37-47.

Fredriksson-Ahomaa, M., Niskanen, T., Bucher, M., Stolle, A. \& Korkeala, H. (2003). Different Yersinia enterocolitica 4: O3 genotypes found in pig tonsils in Southern Germany and Finland. Syst Appl Microbiol 26, 132-137.

Iteman, I., Guiyoule, A. \& Carniel, E. (1996). Comparison of three molecular methods for typing and subtyping pathogenic Yersinia enterocolitica strains. J Med Microbiol 45, 48-56.

Jones, T. F., Buckingham, S. C., Bopp, C. A., Ribot, E. \& Schaffner, W. (2003). From pig to pacifier: chitterling-associated yersiniosis outbreak among black infants. Emerg Infect Dis 9, 1007-1009.

Kapperud, G., Nesbakken, T., Aleksic, S. \& Mollaret, H. H. (1990). Comparison of restriction endonuclease analysis and phenotypic typing methods for differentiation of Yersinia enterocolitica isolates. J Clin Microbiol 28, 1125-1131.

Korte, T., Fredriksson-Ahomaa, M., Niskanen, T. \& Korkeala, H. (2004). Low prevalence of pathogenic Yersinia enterocolitica in sow tonsils. Foodborne Pathog Dis 1, 45-52.

Ostroff, S. M., Kapperud, G., Hutwagner, L. C., Nesbakken, T., Bean, N. H., Lasse, J. \& Tauxe, R. V. (1994). Sources of sporadic Yersinia enterocolitica infections in Norway: a prospective casecontrol study. Epidemiol Infect 112, 133-141.

Tauxe, R. V., Vandepitte, J., Wauters, G., Martin, S. M., Goossens, V., de Moll, P., van Noyen, R. \& Thiers, G. (1987). Yersinia enterocolitica infections and pork: the missing link. Lancet i, 1129-1132. 